\title{
PTU-035 UTILITY OF ENDOSCOPIC ULTRASOUND AND FINE NEEDLE ASPIRATION IN THE DIAGNOSIS OF PANCREATIC NEUROENDOCRINE TUMOURS
}

doi:10.1136/gut.2011.239301.163

J S Leeds, $1^{1^{*}} \mathrm{M}$ M Nayar, ${ }^{1}$ V Wadehra, ${ }^{2}$ K E Oppong ${ }^{1}$ HPB endoscopy, Freeman Hospital, Newcastle Upon Tyne, UK; ${ }^{2}$ Department of Cytology, Freeman Hospital, Newcastle Upon Tyne, UK

Introduction Endoscopic ultrasound (EUS) is used to stage and sample lesions throughout the gastrointestinal tract and is very helpful in assessing pancreatic masses. Neuroendocrine tumours (NETs) of the pancreas account for $1-2 \%$ of mass lesions but require differentiation from carcinomas as the management and prognosis are different. We aimed to assess the utility of EUS and fine needle aspiration cytology (FNA) in the diagnosis of NETs in our unit.

Methods Patients are prospectively added to the EUS FNA database when attending for EUS and the period April 2003April 2010 was examined. All resected lesions were examined for EUS and FNA findings and patient age and gender, lesion site, EUS characteristics and FNA findings were recorded. Sensitivity, specificity, positive and negative predictive value and accuracy were calculated. 
Results 829 EUS FNA procedures (793 patients) were for a solid lesion of which 237 had definitive histology. 34 (4.3\%) patients (mean age 59.7years, 19 males) had a NET with the majority (31/34) being found in the head of the pancreas. All lesions were amenable to FNA but 10/34 lesions yielded inadequate material for cytological analysis (all of which were prior to in room cytological support). The number of passes in adequate samples was higher than in inadequate samples (median 3 vs 2, p=0.058). The sensitivity, specificity, positive, predictive value, negative predictive value and accuracy of EUS and FNA for detecting pancreatic NETs were 0.71, 0.99, 0.77, 0.99 and 0.95 respectively.

Conclusion EUS and FNA is a helpful modality for assessing and sampling potential pancreatic NETs with a positive sample correlating highly with histology. The low sensitivity in the present study may be related to inadequate samples which could be improved by increasing the number of passes into the lesion and in room cytological support.

Competing interests None.

Keywords endoscopic ultrasound, fine needle aspiration, neuroendocrine tumour. 